\title{
ESCORPIONES (ARACHNIDA: SCORPIONES) DEPOSITADOS EN LA COLECCIÓN ARACNOLÓGICA DEL MUSEO NACIONAL DE HISTORIA NATURAL DE SANTO DOMINGO
}

\author{
Solanlly Carrero Jiménez ${ }^{1}$ y Gabriel de los Santos ${ }^{2}$ \\ Museo Nacional de Historia Natural de Santo Domingo (MNHNSD). Calle César Nicolás Penson, \\ Plaza de la Cultura, Santo Domingo, República Dominicana. \\ 1's.carrero@museohistorianatural.gov.do²g.delossantos@museohistorianatural.gov.do
}

\section{RESUMEN}

La colección de escorpiones del MNHNSD contiene 255 especímenes, correspondientes a las familias Buthidae (4 géneros), Scorpionidae (Diplocentrinae, 2) y Hemiscorpiidae (Hormurinae, 1). Centruroides Marx, 1890, es el género mejor representado, con cinco (5) especies y 124 especímenes. La mayor parte de los especímenes procede de la Región Sur de la República Dominicana. Se presenta la lista de las especies depositadas en la colección.

Palabras clave: colección aracnológica, escorpiones, Museo Nacional de Historia Natural de Santo Domingo, República Dominicana.

\section{ABSTRACT}

The MNHNSD collection of scorpions holds 255 specimens belonging to the families Buthidae (4 genera), Scorpionidae (Diplocentrinae, 2) y Hemiscorpiidae (Hormurinae, 1). Centruroides Marx, 1890, is the best represented genus with five species and 124 specimens. Most of specimens have been collected in the southwestern region of the Dominican Republic. A checklist of the species deposited in the collection is presented.

Key words: arachnological collection, scorpions, Museo Nacional de Historia Natural de Santo Domingo, Dominican Republic.

En las Antillas, el Orden Scorpiones está representado por una alta diversidad de especies que pertenecen a cuatro familias (Buthidae, Chactidae, Hemiscorpiidae, Scorpionidae), 15 géneros y 130 especies (de Armas, 2009), la mayoría de ellas limitadas a una o muy pocas islas. Para La Hispaniola se registran tres familias (Buthidae, Diplocentridae y Liochelidae) y 36 especies, correspondientes a ocho (8) géneros; 34 de las especies son endémicas de la isla, mientras que 30 de estas solo están presentes en la República Dominicana (Perez-Gelabert, 2008).

Los resultados que se presentan fueron obtenidos como parte del proceso curatorial de la referida colección (i.e., actualización de la información taxonómica, determinación de especímenes y catalogación de la colección). Esta nota tiene por objetivo presentar la situación actual de la colección de escorpiones del MNHNSD. El ordenamiento taxonómico se hizo siguiendo a Fet y Soleglad (2005) y para las sinonimias a Teruel (2005).

La colección posee 121 lotes, conteniendo 255 especímenes pertenecientes a tres (3) familias, seis (6) géneros y 15 especies. Buthidae es la familia mejor representada con 222 especímenes (85\% de la colección). En cuanto a cantidad de especies y de especímenes, Centruroides Marx, 1890 es el género mejor representado, con cinco (5) y 124, respectivamente. El registro más antiguo de la colección es un ejemplar de Centruroides bani Armas y Marcano, 1987, colectado el 13-VII-1984 por J. A Ottenwalder en Guayabal, Postrer Río, provincia Independencia. 
De las 30 especies de escorpiones endémicas de la República Dominicana, ocho (8) están presentes en la colección, para un 27\%. El 59\% de los especímenes fue colectado en la Región Sur del país: provincias Azua, Bahoruco, Barahona, Elías Piña, Independencia, Pedernales, Peravia, San Cristóbal, San José de Ocoa y San Juan; lo que se traduce en necesarias colectas en las regiones norte y este del país.

En esta colección también se encuentran tres (3) especies procedentes de Cuba, estas son: Centruroides gracilis (Latreille, 1804), Centruroides guanensis Franganillo, 1930 y Cazierius gundlachii Karsch, 1880, donadas por la Academia de Ciencias de Cuba.

\section{LISTADE LAS ESPECIES DE ESCORPIONES DEPOSITADAS EN LACOLECCIÓNDEL MUSEO NACIONAL DE HISTORIA NATURAL DE SANTO DOMINGO}

\section{BUTHIDAE}

Centruroides Marx, 1890

Centruroides bani Armas \& Marcano, 1987

Centruroides gracilis (Latreille, 1804)

Centruroides guanensis Franganillo, 1930

Centruroides marcanoi Armas, 1981

Centruroides tenuis (Thorell, 1876)

=Centruroides nitidus (Thorell, 1876)

=Centruroides nitidus taino Armas \& Marcano, 1987

Centruroides sp.

Microtityus Kjellesvig-Waering, 1966

Microtityus sp.

Rhopalurus Thorell, 1876

Rhopalurus princeps (Karsch, 1879)

Rhopalurus sp.

Tityus C. L. Koch, 1836

Tityus crassimanus (Thorell, 1876)

Tityus quisqueyanus Armas, 1982

Tityus sp.

\section{SCORPIONIDAE: DIPLOCENTRINAE}

Cazierius Francke, 1978

Cazierius gundlachii Karsch, 1880

Cazierius politus (Pocock, 1898), Armas \& Marcano, 1987

Heteronebo Pocol, 1899

Heteronebo dominicus Armas, 1981

= Cazierius dominicus Armas, 1981

HEMISCORPIIDAE: HORMURINAE

Opisthacanthus Peters, 1861

Opisthacanthus lepturus (Palisot de Beauvois, 1805)

=Opisthacanthus laevicauda (Thorell, 1877) 


\section{LITERATURA CITADA}

Armas, L. F. de. 2009. Scorpions. The Antillean or West Indian fauna [Internet]. Version 19. Knol. 2009. Mar 1. http://knol.google.com/k/luis-f-de-armas/scorpions-the-antillean-orwest-indian/kbg2jg4ueep $9 / 6$

Fet, V. y M. E. Soleglad. 2005. Contributions to scorpions systematics. I. On recent changes in high-level taxonomy. Euscorpius. December 2005, no. 31.

Perez-Gelabert, D. E. 2008. Arthropods of Hispaniola (Dominican Republic and Haiti): A checklist and bibliography. Zootaxa 1831: 1-530.

Teruel, R. 2005. Nuevos datos sobre la taxonomía, distribución geográfica y ecología de los escorpiones de la República Dominicana (Scorpiones: Liochelidae, Scorpionidae, Buthidae). Boletín de la Sociedad Entomológica Aragonesa, 36, 165-176. 